\title{
EVALUACIÓN DEL CRECIMIENTO DE FRíJOL (Phaseolus vulgaris L.) CV ICA CERINZA, BAJO ESTRÉS SALINO
}

\section{EVALUATION OF BEAN (Phaseolus vulgaris L.) CV 'ICA CERINZA' GROWTH, UNDER SALT STRESS}

\author{
Wilmer Alejandro Quintana-Blanco ${ }^{1}$, Elberth Hernando Pinzón-Sandoval ${ }^{2}$, David Fernando Torres ${ }^{3}$
}

\begin{abstract}
${ }^{1}$ Estudiante Ingeniería Agronómica. Universidad Pedagógica y Tecnológica de Colombia, Tunja, Boyacá. Av. Central del Norte, e-mail: wilquintana61@hotmail.com; ${ }^{2}$ Ing. Agrónomo, M.Sc. Fisiología Vegetal, Grupo de Investigaciones Agrícolas. Universidad Pedagógica y Tecnológica de Colombia UPTC. Av. Central del Norte. Tunja, Boyacá, e-mail: elberth02@gmail. com; ${ }^{3}$ Ing. Agrónomo, cM.Sc. Fisiología Vegetal. UPTC - Universidad de Ciencias Aplicadas y Ambientales U.D.C.A, e-mail: mollisol89@hotmail.com
\end{abstract}

Rev. U.D.C.A Act. \& Div. Cient. 19(1): 87-95, Enero- Junio, 2016

\section{RESUMEN}

El fríjol común (Phaseolus vulgaris L.) es uno de los productos más importantes en la alimentación humana. Además, se encuentra ampliamente distribuido en las regiones tropicales y subtropicales del mundo. En la actualidad, la mayoría de las zonas aptas para la agricultura presentan problemas de salinización, ya sea por la condición natural del suelo o por acción antrópica. El frijol es sensible a la salinidad, ya que puede reducir su rendimiento hasta en un $50 \%$. El objetivo de esta investigación fue evaluar el comportamiento del cultivar Ica Cerinza, sometido a diferentes concentraciones de $\mathrm{NaCl}$, en condiciones de invernadero; los tratamientos para generar estrés por salinidad fueron $0,20,40$ y $60 \mathrm{mM}$ de $\mathrm{NaCl}$. Se utilizó un diseño completamente aleatorizado (DCA). Se evaluaron parámetros fisiológicos, como resistencia estomática, contenido total de clorofila, área foliar, peso fresco y seco de órganos, grosor de la hoja y longitud de raíz. A medida que se aumentó la dosis de $\mathrm{NaCl}$, el contenido total de clorofila disminuyó y la resistencia estomática aumentó, lo que se vio reflejado en el escaso crecimiento y acumulación de materia seca y fresca, por parte de la planta; sin embargo, el cultivar mostró una tolerancia a una condición moderada de salinidad, ya que generó respuestas de tipo morfológico, con lo que evitó una disminución drástica del crecimiento en su etapa vegetativa.

Palabras clave: Salinidad, comportamiento fisiológico, leguminosa, crecimiento.

\section{SUMMARY}

The common bean (Phaseolus vulgaris L.) is one of the most important products for human feeding. In addition, bean cultivations is widely distributed in tropical and subtropical regions. At present most areas suitable for agricultural purposes have salinization problems either by natural soil conditions or because of human action. The common bean is sensitive to salinity, as this condition could reduce yield up to $50 \%$. The objective of this research was to evaluate the behavior of the cultivar Ica Cerinza, subjected to different concentrations of $\mathrm{NaCl}$ under greenhouse conditions. The treatment to generate salt stress were $0,20,40$ and $60 \mathrm{mM}$ of $\mathrm{NaCl}$. The experimental design used was completely randomized. Physiological parameters such as, stomatal resistance, chlorophyll content, leaf area, fresh and dry weight of organs, leaf thickness and root length were evaluated. Result showed that as $\mathrm{NaCl}$ dose was increased, chlorophyll content decreased and stomatal resistance increased, which was reflected in scares growth and accumulation of dry and fresh matter from the plant. However the cultivar showed a tolerance to a moderate salinity condition, since it generated morphological type responses, which avoided a drastic reduction of increase during the vegetative growth stage.

Key words: Salinity, physiological behavior, leguminous, growth.

\section{INTRODUCCIÓN}

El fríjol común (Phaseolus vulgaris L.) es la especie más importante para el consumo humano, entre las leguminosas de grano alimenticias y tiene gran importancia en la dieta de la población colombiana, por su alto contenido proteico y de minerales esenciales, siendo un producto clave en la seguridad alimentaria de la población (Delgado et al. 2013). Presenta otras características, que hacen ventajoso su consumo, desde el punto de vista nutricional, como su elevado 
contenido de lisina, fibra alimentaria y presencia de vitaminas del complejo B. América Latina es el mayor productor y consumidor, con más del $45 \%$ de la producción mundial (CNPAF- EMBRAPA, 2003; Arias et al. 2007). En Colombia, para el primer semestre de 2015, la producción de fríjol ascendió a 62.974t, de la cual, el departamento de Boyacá participó con 1.189 t, en un área de 820 ha y una producción promedio de 1,5t.ha ${ }^{-1}$ frijol seco (FENALCE, 2016).

La salinidad en los suelos es un problema para la agricultura del mundo, uno de los factores que más limita la productividad de los cultivos (Gouia et al. 1994); en Colombia, aproximadamente el $1 \%$ de los suelos están afectados por problemas de este tipo (FAO, 2000). Las zonas susceptibles a la salinización en el país abarcan gran parte de la región Caribe, los valles interandinos (ríos Magdalena y Cauca) y los altiplanos, donde actualmente se concentra la producción agrícola del país (MAVDT, 2004). En Boyacá, se consideran que existen 23.500 ha con susceptibilidad a procesos de salinización, debido a la formación de suelos sulfatados, ácidos salinos en los valles de Tundama, Sugamuxi, Ubaté y Cucunubá (Castro \& Gómez, 2015).

Las sales tienen efectos adversos sobre las propiedades físicas, químicas y microbiológicas del suelo (Gili et al. 2004). Varias son las causas vinculadas a los procesos de salinización, entre las cuales, es posible citar el excesivo uso de fertilizantes, calidad del agua de riego, falta de obras de ingeniería, como los drenajes y ampliación de la frontera agrícola hacia zonas de vegetación nativa (Coca et al. 2012).

El estrés salino ocurre por altas concentraciones de sodio $\left(\mathrm{Na}^{+}\right)$y cloro $(\mathrm{Cl})$ en la solución nutritiva, que induce serias alteraciones en el metabolismo de la planta, que repercute en su crecimiento y desarrollo (Hasegawa et al. 2002). Sumado a lo anterior, conduce a otros tipos de estrés, como el ocasionado por el déficit hídrico, debido al efecto osmótico y denominado mecanismo no específico de la salinización, que se torna en uno de los factores que reducen la tasa de fijación de dióxido de carbono $\left(\mathrm{CO}_{2}\right)$, con el que se afectan los procesos fotosintéticos de las plantas (Sudhir \& Murthy, 2004).

El fríjol común es sensible a la salinidad, ya que puede reducir su rendimiento hasta en un $50 \%$, cuando se presenta una conductividad eléctrica (CE) del suelo, mayor o igual a $2 \mathrm{dS} / \mathrm{m}$, equivalente a $20 \mathrm{mM} \mathrm{NaCl}$ (Bayuelo-Jiménez et al. 2002). Una elevada concentración salina del suelo o el sustrato afecta también, considerablemente, la tasa de germinación, debido a la disminución del potencial hídrico, con lo que se reduce la disponibilidad de agua, necesaria para imbibición que requiere las semilla para germinar (Kaymakanova et al. 2008). La reducción de la germinación es diferencial entre cultivares, como lo indican Can Chulim et al. (2014), quienes encontraron disminución de 25,3 hasta
$41,7 \%$, cuando se presentó una conductividad eléctrica de $6,3 \mathrm{dS} \mathrm{m}^{-1}$.

Se ha encontrado que algunas especies del genero Phaseolus presentan mecanismos de tipo genético y morfológico, que les permiten tolerar la salinidad y los efectos adversos, ocasionados por el déficit hídrico, como respuesta al estrés por salinidad (Lizana et al. 2006). La reducción del impacto de la salinidad sobre el crecimiento de los vegetales, bajo un modelo convencional, requiere, con frecuencia, el uso de enmiendas aplicadas al suelo (Casierra \& García, 2006) o de tecnologías, como el uso de biofertilizantes, biopolímeros o aplicación de campos electromagnéticos (Zúñiga et al. 2011), que incrementan los costos de producción y no son totalmente efectivas. Por lo anterior, la investigación tuvo como finalidad, la evaluación de parámetros fisiológicos en etapa vegetativa del cultivar de fríjol, de mayor importancia en la región de Boyacá, con el fin de conocer su respuesta al estrés, causado por salinidad.

\section{MATERIALES Y MÉTODOS}

El trabajo, se desarrolló en el invernadero de vidrio de la Universidad Pedagógica y Tecnológica de Colombia, sede Tunja, ubicada a $5^{\circ} 32^{\prime \prime} 25^{\prime \prime} \mathrm{N} 73^{\circ} 21^{\prime} 41^{\prime \prime} \mathrm{O}$, con una altura de 2.691 msnm, humedad relativa promedio de $80 \%$ y temperatura promedio de $26^{\circ} \mathrm{C}$.

Para determinar el efecto de la salinidad por $\mathrm{NaCl}$ sobre el crecimiento en plantas de fríjol, se seleccionó el cultivar Ica Cerinza, que es una material regional para zonas de clima frío y frío moderado, de tipo arbustivo, uniforme en cuanto a tamaño, número de granos y distribución en la planta, grano de tipo radical de tamaño mediano y color rojo oscuro (Ríos \& Quirós, 2002; Astudillo \& Blair, 2008), que se cultiva comúnmente en Boyacá. Las semillas fueron obtenidas de FENALCE, con el fin de garantizar las características genéticas del cultivar; posteriormente, se sembraron en materas plásticas, con capacidad para $2 \mathrm{~kg}$. El sustrato utilizado fue suelo negro, al cual, se le realizó análisis de laboratorio, para descartar que presentara alta conductividad o elevado contenido de sodio (Tabla 1).

Las plantas, se establecieron en el primer semestre del 2015. La condición de estrés, se simuló mediante la aplicación de Cloruro de Sodio $(\mathrm{NaCl})$, siendo los tratamientos: T0: $\sin$ aplicación de $\mathrm{NaCl}$; T1: $20 \mathrm{mM}$ de $\mathrm{NaCl}$; T2: $40 \mathrm{mM}$ de $\mathrm{NaCl}$ y $60 \mathrm{mM}$ de $\mathrm{NaCl}$, cada uno con tres réplicas, para un total de 12 unidades experimentales, cada una compuesta por dos plantas. $\mathrm{El} \mathrm{NaCl}$, se diluyó y aplicó a cada matera, con una frecuencia de riego de 2 días, en volumen de $100 \mathrm{~mL}$ por planta, durante seis semanas. Las aplicaciones comenzaron cuando las plantas se encontraron en estadio V14 hasta el estadio V19, con el fin de garantizar que estuvieran comple- 
tamente establecidas y presentaran homogeneidad (Feller et al. 1995).

Los parámetros fisiológicos no destructivos evaluados fueron: clorofila total, relacionada con el contenido de nitrógeno de la hoja, con un clorofilómetro Minolta SPAD 502 plus: se tomó por planta un total de 10 mediciones, para generar un promedio por cada unidad experimental; resistencia estomática, mediante un porómetro Decagon Devices SC-1: se realizó la medición en hojas del tercio superior. Las mediciones, se efectuaron semanalmente, posterior al inicio de los tratamientos.

Tabla 1. Propiedades fisicoquímicas del sustrato.

\begin{tabular}{|c|c|}
\hline Propiedades & Resultado \\
\hline Densidad aparente & $1,2 \mathrm{~g} \mathrm{~cm}^{-3}$ \\
\hline Densidad real & $2,3 \mathrm{~g} \mathrm{~cm}^{-3}$ \\
\hline Porosidad & $48 \%$ \\
\hline Conductividad eléctrica & $0,2 \mathrm{dS} \mathrm{m}{ }^{-1}$ \\
\hline $\mathrm{pH}$ & 4,8 \\
\hline $\mathrm{M} . \mathrm{O}$ & $4,8 \%$ \\
\hline $\mathrm{Na}$ & $0,88 \mathrm{cmol} \mathrm{kg}^{-1}$ de suelo \\
\hline $\mathrm{Ca}$ & $1,58 \mathrm{cmol} \mathrm{kg}^{-1}$ de suelo \\
\hline $\mathrm{Mg}$ & $0,27 \mathrm{cmol} \mathrm{kg}^{-1}$ de suelo \\
\hline
\end{tabular}

Para la evaluación de los parámetros fisiológicos destructivos, se determinó el peso fresco total y peso seco total, secando las muestras en estufa de secado, durante $48 \mathrm{~h}$, momento en el que alcanzaron peso constante; las muestras fueron pesadas, con una balanza electrónica Acculab VIC 612 de 0,01 $\mathrm{g}$ de precisión y secadas en una estufa de secado Memmert; grosor de hoja, por medio de un calibrador digital Mitutoyo precisión $\pm 0,05 \mathrm{~mm}$ y área foliar, con un medidor LICOR 2000. Lo anterior, se procedió, luego de seis semanas de aplicación de tratamientos, momento en el que terminó el ensayo.

Los datos obtenidos fueron sometidos a pruebas de normalidad y de homogeneidad de varianza, mediante las pruebas de Shapiro-Will y Levene, respectivamente. Comprobados los supuestos, se realizó análisis de varianza; las variables que mostraron diferencias estadísticas fueron sometidas a pruebas de comparación de medias de Tukey $(P \leq 0,05)$. Los análisis se formalizaron con el programa estadístico SAS v.9.2e SAS Institute Inc., Cary,NC.

\section{RESULTADOS Y DISCUSIÓN}

El contenido de clorofila total presentó diferencias significativas $(P \leq 0,05)$, entre las dosis utilizadas. Se pudo observar que, luego de dos semanas de aplicación de la condición de estrés, los contenidos de clorofila comenzaron a descender en los tratamientos con las mayores dosis (Figura 1); mientras mayor estrés se presentó en las plantas a causa del $\mathrm{NaCl}$, menor fue el contenido de clorofila. El método utilizado fue de tipo no destructivo y el que, como mencionan Fang et al. (2010), se puede utilizar para monitorear el contenido de $\mathrm{N}$ en la hoja. La salinidad generó senescencia foliar, acompañada de removilización de nutrientes, como nitrógeno de las hojas maduras a las jóvenes, debido a la baja absorción de iones, como el nitrato, tal como lo reportan McCue \& Hanson (1990). Existen investigaciones que coinciden en que el aumento en la concentración de $\mathrm{Na}^{+}$en la solución del suelo y de la planta afectan la concentración de pigmentos fotosintéticos, debido, principalmente, a la destrucción de los cloroplastos y a un aumento de la actividad de la enzima clorofilasa, dando paso a la degradación de las clorofilas (Flowers \& Yeo, 1986; Appels \& Lagudah, 1990). Los resultados encontrados en el presente estudio coinciden con algunas investigaciones, en las que se indica que cultivares de $P$. vulgaris disminuyen el contenido de clorofila al aumentar las dosis de $\mathrm{NaCl}$, por encima de $20 \mathrm{mM}$ (Jaramillo et al. 2009).

En cuanto a la resistencia estomática, se observaron diferencias significativas $(\mathrm{P} \leq 0,05)$ entre las dosis de $\mathrm{NaCl}$ apli-

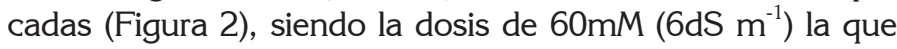
presentó el mayor valor, con una resistencia estomática de $721 \pm 66,06 \mathrm{~m}^{2} \mathrm{~s} \mathrm{~mol}^{-1}$, luego de 28 días de inicio de aplicación del tratamiento. Se pudo observar que este parámetro aumentó, de manera notoria, con cualquiera de las dosis aplicadas, mientras que el tratamiento testigo se mantuvo estable, a través del tiempo. La condición de estrés genera que 
desde la raíz se presente una síntesis de ácido abscísico (ABA), como respuesta temprana, que produce cambios fisiológicos locales, como reducción de la conductividad hidráulica y a distancia, cierre estomático (Hartung et al. 2002).

Una de las respuestas primarias de las plantas al estar sometidas a condiciones de estrés es el cierre estomático, que genera un bloqueo directo de la fotosíntesis, debido a que se limita la entrada de $\mathrm{CO}_{2}$, sustrato necesario para el ciclo de Calvin. Esto ocasiona un bloqueo de la fase foto, por la nula demanda del poder reductor y ATP, dando como resultado la producción de oxígeno, con un consecuente daño de las membranas de los cloroplastos y posterior desintegración de los mismos (Appels \& Lagudah, 1990). Lo anterior correlaciona con la disminución en los niveles de clorofila, observados en la presente investigación (Figura 1).

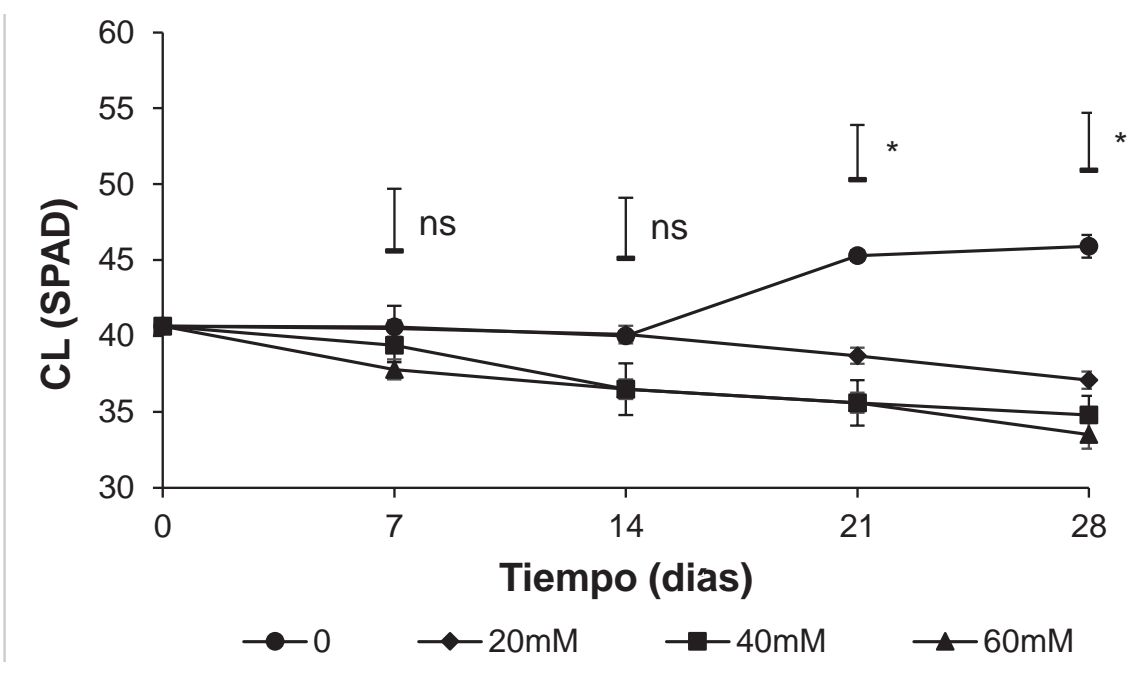

Figura 1. Comportamiento de la clorofila total en fríjol (Phaseolus vulgaris L.) cv Ica Cerinza, sometido a diferentes dosis de $\mathrm{NaCl}$, en condiciones controladas. Barras verticales en los tratamientos indican error estándar $(n=4)$. Barras en cada punto de muestreo indica la diferencia mínima significativa, según la prueba de Tukey $(P \leq 0,05)$, ns: no existen diferencia estadística, *: diferencias significativas al $95 \%$.

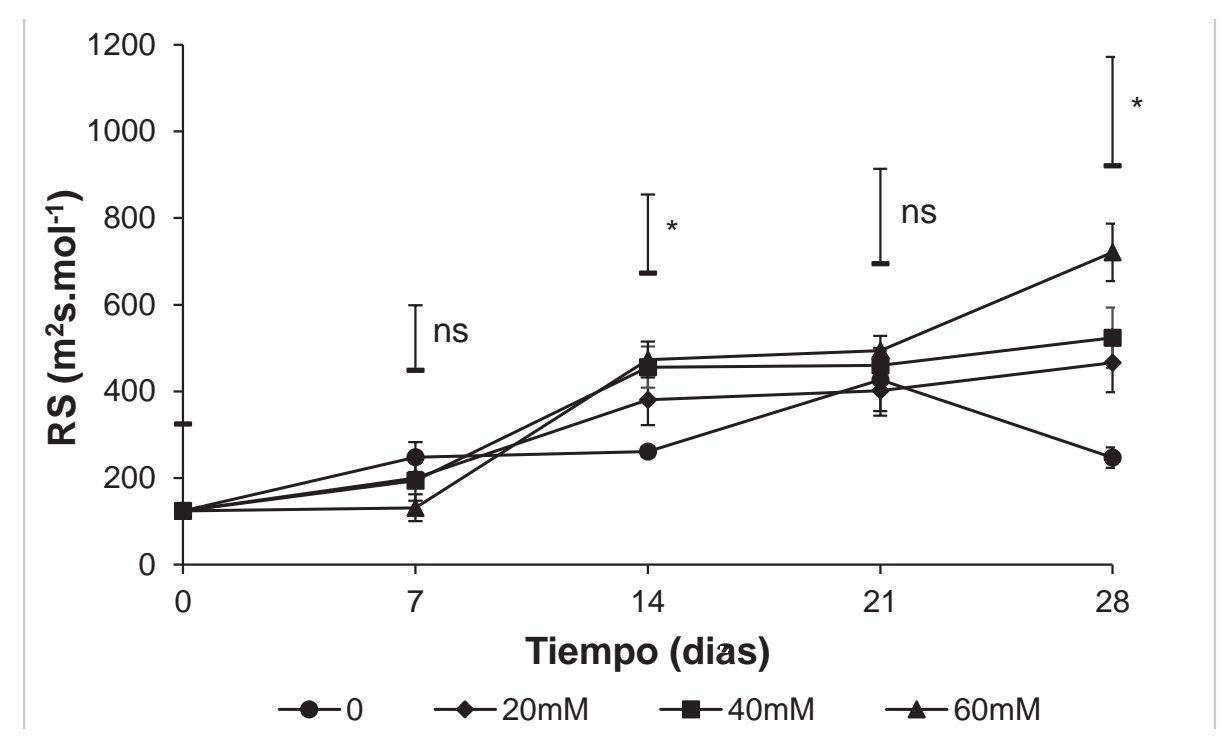

Figura 2. Comportamiento de la resistencia estomática en fríjol (Phaseolus vulgaris L.) cv Ica Cerinza, sometido a diferentes dosis de $\mathrm{NaCl}$, en condiciones controladas. Barras verticales en los tratamientos indican error estándar $(\mathrm{n}=4)$. Barras en cada punto de muestreo indica la diferencia mínima significativa, según la prueba de Tukey $(\mathrm{P} \leq 0,05)$, ns: no existen diferencia estadística, *: diferencias significativas al 95\%. 
El estrés por salinidad también tiene un efecto importante en el proceso de transpiración, relacionado con el incremento de la resistencia al flujo de agua hacia el interior de la planta, dependiente de la regulación estomática (Tabatabaei, 2006). Los resultados indican que el fríjol es una especie sensible al estrés por salinidad. Algunos autores afirman que este es un cultivo que sufre daños considerables, a partir de 1dS $\mathrm{m}^{-1}$, equivalente a $10 \mathrm{mM}$ de $\mathrm{NaCl}$ (Maas, 1990). La salinidad altera las relaciones hídricas y el intercambio gaseoso de especies cultivadas y silvestres del género Phaseolus (BayueloJiménez et al. 2002).

A

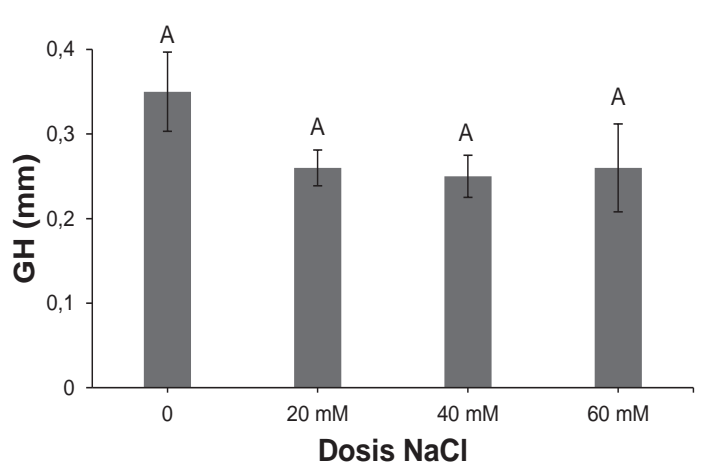

No se presentaron diferencias estadísticas para el grosor de hoja y la longitud de raíz (Figura 3A); sin embargo, el tratamiento sin aplicación de $\mathrm{NaCl}$ presentó un grosor de hoja de 0,35 $\pm 0,04 \mathrm{~mm}$, mientras que los tratamientos con algún tipo de aplicación de $\mathrm{NaCl}$, mostraron valores entre $0,25 \pm 0,02 \mathrm{~mm}$ y $0,26 \pm 0,05 \mathrm{~mm}$, siendo inferiores al obtenido en el testigo. Las plantas sometidas a la condición de estrés presentan impedimento para mantener un buen estatus hídrico, debido al cierre estomático y el desajuste osmótico, reflejado en una menor cantidad de agua almacenada en órganos, como la hoja.

B

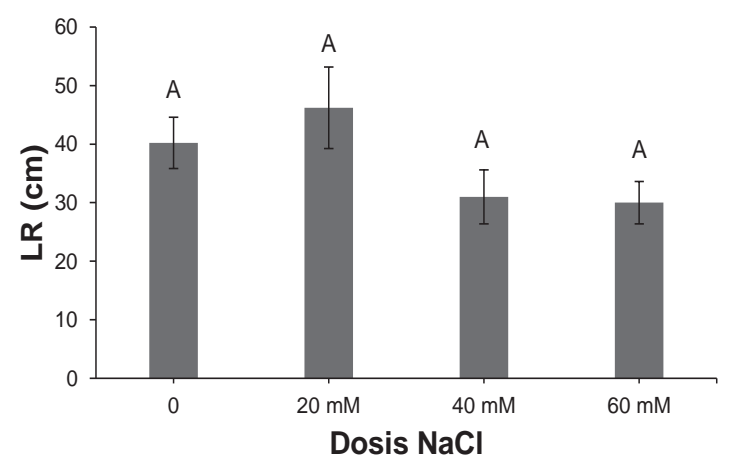

Figura 3. A) Grosor de hoja; B) Longitud de raíz, en fríjol (Phaseolus vulgaris L.) cv Ica Cerinza, sometido a diferentes dosis de $\mathrm{NaCl}$, en condiciones controladas. Tratamientos seguidos de letras distintas presentan diferencias significativas, según la prueba de Tukey $(P \leq 0,05)$, barras verticales indican error estándar $(n=4)$.

Por su parte, la variable longitud de raíz presentó un valor máximo de 46,2 $\pm 6,9 \mathrm{~cm}$, que correspondió al tratamiento de $20 \mathrm{mM}$ de $\mathrm{NaCl}$, mientras en los tratamientos de $40 \mathrm{y}$ $60 \mathrm{mM}$, el tamaño de la raíz se vio afectado de manera notoria, pues se observaron valores de $31 \pm 4,6 \mathrm{~cm}$ y $30 \pm 3,6 \mathrm{~cm}$, respectivamente (Figura $3 \mathrm{~B}$ ). Lo encontrado en esta variable indica que una condición de estrés moderada, dosis de 20mM, generó, como respuesta, un aumento de la longitud de la raíz. Campos et al. (2011) observaron también un aumento en la longitud del sistema radical en cultivares de fríjol sometidos a estrés por $\mathrm{NaCl}$, lo que representó una ventaja en cuanto a la capacidad para la obtención del agua en perfiles subsuperficiales del suelo, lo que contribuye a enfrentar el estrés osmótico, impuesto por las sales. Por el contrario, Velázquez et al. (2002) hallaron que al aplicar sales sódicoalcalinas inhibieron el crecimiento de la raíz, debido a los procesos hidrolíticos y el $\mathrm{Na}^{+}$, que actúan a nivel de meristemos, destruyendo las células vegetales e inhibiendo la síntesis de hormonas, lo que limita el crecimiento de la planta.

Por su parte, el área foliar, peso fresco total y peso seco total presentaron diferencias significativas entre tratamien- tos, según la prueba de Tukey $(P \leq 0,05)$ (Figura 4 y 5). El tratamiento de $20 \mathrm{mM}$ de $\mathrm{NaCl}$ mostró el mayor valor para la variable área foliar, con $182,6 \pm 18,4 \mathrm{~cm}^{2}$ y reveló diferencias significativas frente a los tratamientos 3 y 4 , en los cuales, se evidenciaron los menores valores, con 88,2 $\pm 16,1$ y $92,61 \pm 18,7 \mathrm{~cm}^{2}$, respectivamente. Lo observado en el tratamiento $20 \mathrm{mM}$, se puede deber a una respuesta morfológica por parte de este cultivar, para buscar sobreponerse a la condición de salinidad, bajo una concentración moderada de $\mathrm{NaCl}$; esta respuesta indica que la planta generó mayor superficie de tejido foliar, con el fin de buscar una dilución por crecimiento (Campos et al. 2011). Los mismos autores indican que en cultivares de $P$. vulgaris, sometidos a condiciones de salinidad, redujeron el área foliar, así como otros componentes de crecimiento, debido, tal vez, al desajuste osmótico o al efecto tóxico, ocasionado por el $\mathrm{NaCl}$, en concentraciones de $40 \mathrm{mM}$. Lo anterior correlaciona con lo observado en los tratamientos 3 y 4 ( 40 y $60 \mathrm{mM})$.

Aschan \& Pfanz (2003) indican que el crecimiento de las plantas es el resultado directo de una masiva y rápida expansión de las células jóvenes producidas por las divisiones 
meristemáticas; no obstante, la expansión celular, tanto de la raíz como del área foliar, puede ser inhibida por la salinidad, cuando se presenta en concentraciones altas, como lo reporta Ashraf \& Leary (1996), que concuerda con lo observado en el presente estudio.
Se presentaron diferencias significativas en cuanto a las variables peso fresco y seco de parte aérea y peso fresco de raíz. El tratamiento de $20 \mathrm{mM}$ fue el que mostró los mayores valores, mientras que en los tratamientos 3 y 4 , se evidenció una disminución notable, en cuanto a los pesos frescos y secos de raíz y parte aérea (Figura 5).

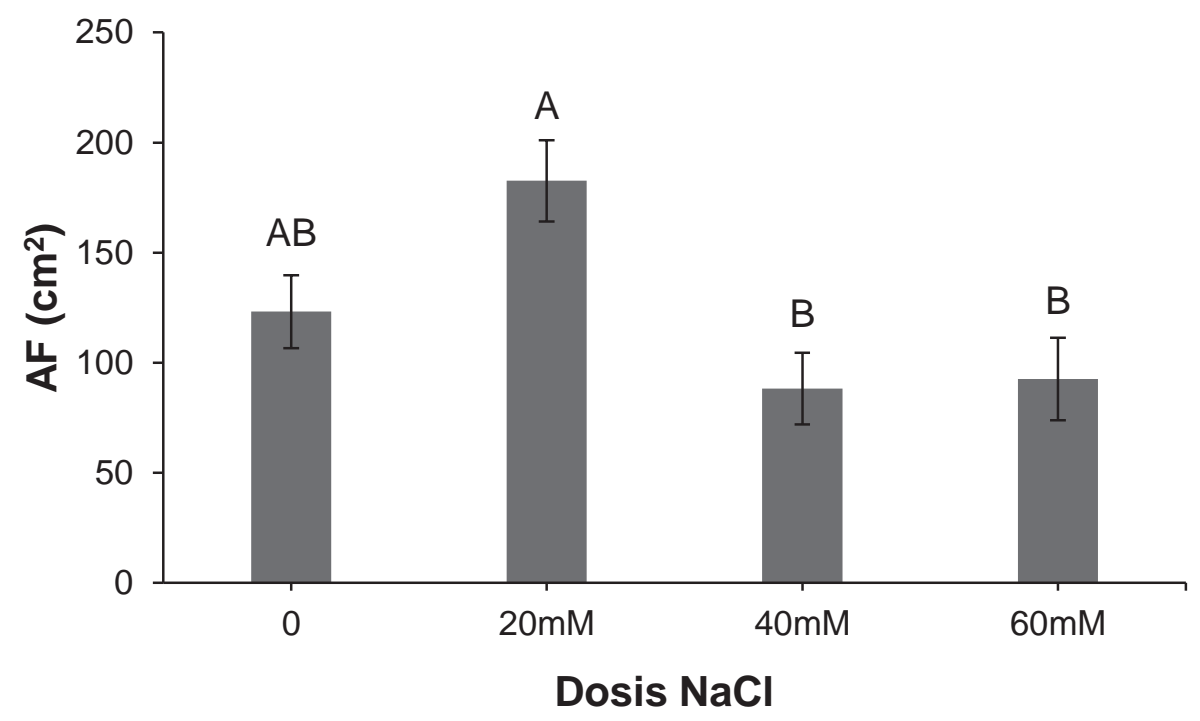

Figura 4. Área Foliar en plantas de fríjol (Phaseolus vulgaris L.) cv Ica Cerinza, sometido a diferentes dosis de $\mathrm{NaCl}$, en condiciones controladas. Tratamientos seguidos de letras distintas presentan diferencias significativas, según la prueba de Tukey $(P \leq 0,05)$, barras verticales indican error estándar $(n=4)$.

A

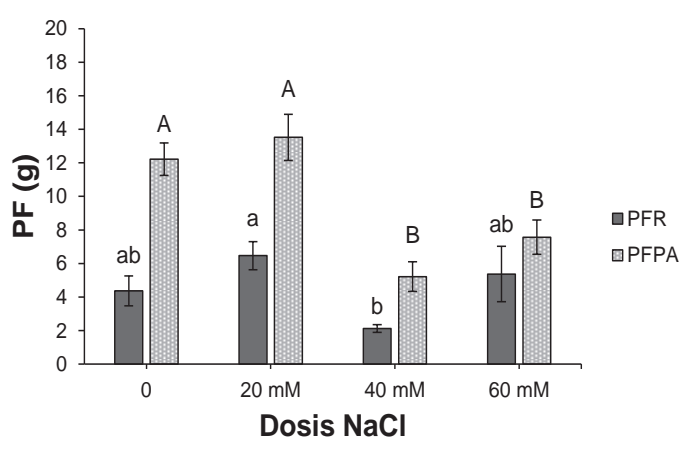

B

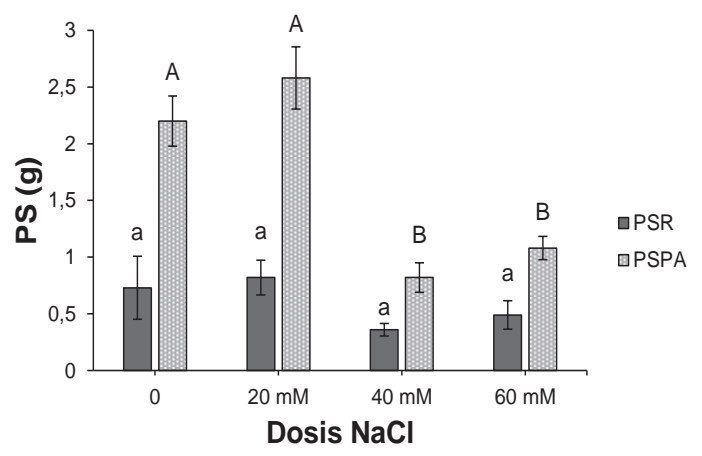

Figura 5. A) PFR: Peso fresco de raíz; PFA: Peso fresco parte aérea; B) PSR: Peso seco de raíz; PSA: Peso seco parte aérea, en frijol (Phaseolus vulgaris L.) cv Ica Cerinza, sometido a diferentes dosis de $\mathrm{NaCl}$, en condiciones controladas. Tratamientos seguidos de letras distintas presentan diferencias significativas, según la prueba de Tukey $(P \leq 0,05)$, barras verticales indican error estándar $(n=4)$. 
La ganancia de peso fresco, se relaciona, principalmente, con la entrada de agua a la planta vía corriente transpiratoria, la cual, se vio afectada, debido a la disminución de la resistencia estomática (Figura 2). Se pudo observar que al generar un aumento en la dosis de $\mathrm{NaCl}(40$ y $60 \mathrm{mM})$, se disminuyó, considerablemente, el crecimiento, tanto de la raíz como de la parte aérea. La reducción del crecimiento de las plantas bajo estrés salino, se atribuye a una alteración en la tasa fotosintética y modificación en el metabolismo de los carbohidratos y su posterior distribución (Argentel et al. 2009). Los mismos autores indican que la producción de biomasa y relación raíz/follaje son criterios comúnmente utilizados, para seleccionar individuos con tolerancia a la salinidad, ya que el efecto inhibitorio, que causa el estrés salino sobre estas variables de crecimiento, es más notable en los cultivares sensibles a la salinidad, que en los tolerantes de la misma especie; esto indica, que el cultivar evaluado, se podría clasificar como sensible, debido a la drástica disminución del peso seco presentada al aumentar la concentración por encima de $20 \mathrm{mM}$ de $\mathrm{NaCl}$.

Kumar (1991) indican que la disminución en la acumulación parte aérea/raíz ha sido muy estudiada, pero que este fenómeno no es general, sino característico de algunas especies; sin embargo, Mano \& Takeda (2001) observaron que las plantas, generalmente, mantienen una proporción característica entre raíz/follaje, cuando esta proporción es afectada; la respuesta inmediata de la planta es disminuir la acumulación de materia seca en ambas partes y, muchas veces, trata de compensar la pérdida, mediante un rápido crecimiento de la parte afectada, para restablecer la proporción raíz/follaje que la caracteriza. Esto correlaciona con lo observado en este trabajo, en que la relación raíz/parte aérea guardó una proporción en cada uno de los tratamientos.

Por su parte, Viswanathan \& Zhu (2003) discuten que el potencial del plasmalema en las células vegetales favorece el transporte pasivo del $\mathrm{Na}^{+}$hacia el interior de las células, especialmente, bajo condiciones de alto contenido de $\mathrm{Na}^{+}$extracelular; sin embargo, el exceso de $\mathrm{Na}^{+}$extracelular entra a la célula, a través del transportador HKT1 y de los canales transportadores de cationes no selectivos, que resulta en una disminución de la relación $\mathrm{K}^{+} / \mathrm{Na}^{+}$en el citosol, que tiene como consecuencia la alteración de los procesos metabólicos, en los cuales, está implicado el $\mathrm{K}^{+} \mathrm{y}$, por tanto, el crecimiento, expresado en términos de acumulación de materia seca, se ve seriamente afectado. Casierra \& García (2006) indican que al someter a condiciones de estrés a plantas de tipo glicófitas, se reduce el crecimiento, debido a que se comprometen algunos de los factores determinantes del proceso fotosintético, como la entrada de $\mathrm{CO}_{2}$ o la disponibilidad de $\mathrm{K}^{+}$.

Se puede concluir, que el cultivar de fríjol Ica Cerinza mostró diferencias significativas, en parámetros fisiológicos, como resistencia estomática, contenido de clorofila total, área foliar y acumulación de materia fresca y seca de parte aérea. La condición de salinidad, a la cual, fue sometido el cultivar, generó un desbalance notable, a nivel de intercambio gaseoso, debido, posiblemente, al cierre estomático, desencadenando problemas en los procesos, como la fotosíntesis y la toma de nutrientes vía flujo en masa, lo que se vio reflejado en el escaso crecimiento y acumulación de materia seca, por parte de la planta; sin embargo, el cultivar reveló una tolerancia a la condición moderada de salinidad, ya que generó respuestas de tipo morfológico, con lo que evitó una disminución drástica del crecimiento en su etapa vegetativa.

Conflicto de intereses: El manuscrito fue preparado y revisado con la participación de todos los autores, quienes declaramos que no existe conflicto de intereses que ponga en riesgo la validez de los resultados presentado.

\section{BIBLIOGRAFÍA}

1. APPELS, A.; LAGUDAH, H.E. 1990. Manipulation of chromosomal segments from wild wheat for the improvement of bread wheat. Austral. J. Plant Physiol. 17:253-266.

2. ARIAS, J.H.; JARAMILLO, M.; RENGIFO, T. 2007. Manual Técnico: Buenas Prácticas Agrícolas (BPA) en la producción de fríjol voluble. CORPOICA - MANA - FAO. C.I. La Selva. Medellín. 168p.

3. ARGENTEL, L.; LÓPEZ, D.; GONZÁLEZ, L.; LÓPEZ, R.; GÓMEZ, E.; GIRÓN, R.; FONSECA I. 2009. Contenido de clorofila e iones en la variedad de trigo harinero Cuba-C-204 en condiciones de estrés salino. Cultivos Tropicales. 30(4):32-37.

4. ASCHAN, G.; PFANZ, H. 2003. Non-foliar photosynthesis-a strategy of additional carbon acquisition. Flora. 198(2):81-97.

5. ASHRAF, M.; LEARY, J.W.O. 1996. Effect of drought stress on growth, water relations, and gas exchange of two lines of sunflower differing in degree of salt tolerance. Int. J. Plant Sci. 157(6):729-732.

6. ASTUDILLO, C.; BLAIR, M. 2008. Contenido de hierro y cinc en la semilla y su respuesta al nivel de fertilización con fósforo en 40 variedades de fríjol colombianas. Agron. Colom. 26(3):471-476.

7. BAYUELO-JIMÉNEZ, J.; DEBOUCK, D; LYNCH, J. 2002. Salinity tolerance in Phaseolus species during early vegetative growth. Crop Science. 42:2184-2192. 
8. CAN CHULIM, Á.; RAMÍREZ, L.; ORTEGA, H.; CRUZ, E.; ROMÁN, D.; SÁNCHEZ, E.; MADUEÑO A. 2014. Germinación y crecimiento de plántulas de Phaseolus vulgaris L. en condiciones de salinidad. Rev. Mex. Ciencias Agr. 5(5):753-763.

9. CAMPOS, G.; GARCÍA, M.; PÉREZ, D.; RAMIS, C. 2011. Respuesta de 20 variedades de carota (Phaseolus vulgaris L.) ante el estrés por $\mathrm{NaCl}$ durante la germinación y en la fase plantular. Bioagro. 23(3):215224.

10. CASIERRA, F.; GARCÍA, N. 2006. Crecimiento y distribución de materia seca en cultivares de fresa (Fragaria sp.) bajo estrés salino. Agron. Colom. 23(1):83-89.

11. CASTRO, H.; GOMEZ, M. 2015. Suelos sulfatados ácidos; el caso del valle alto del rio Chicamocha Boyacá-Colombia. Editorial U.P.T.C. Tunja (Colombia). 238p.

12. CNPAF-EMBRAPA. 2003. Cultivo de feijoeiro. Disponible desde internet en:http://sistemasdeproducao.cnptia. embrapa.br/FontesHTML/Feijao/CultivodoFeijoeiro/ index.htm (con acceso el 15/09/2015).

13. COCA, A.; CARRANZA, C.; MIRANDA, D.; RODRIGUEZ, M. 2012. Efecto del $\mathrm{NaCl}$ sobre los parámetros de crecimiento, rendimiento y calidad de la cebolla de bulbo (Allium cepa L.) bajo condiciones controladas. Rev. Colomb. Cienc. Hortíc. 6(2):196-212.

14. DELGADO, H.; PINZÓN, E.H.; BLAIR, M.; IZQUIERDO, P.C. 2013. Evaluación de líneas de fríjol (Phaseolus vulgaris L.) de retrocruce avanzado entre una accesión silvestre y radical cerinza. Rev. U.D.C.A Act. \& Div. Cient. 16(1):79-86.

15. FANG, L.F.; FENG, L.; SONG, Q.J.; YUAN-S., D.; SU, C.L.; WANG, K. 2010. Investigation of SPAD meterbased indices for estimating rice nitrogen status. Computers Electronics Agricult. 715:560-565.

16. FEDERACIÓN NACIONAL DE CULTIVADORES DE CEREALES Y LEGUMINOSAS -FENALCE-. 2016. Cifras de producción frijol primer semestre de 2015. Disponible desde internet en ww.fenalce.org/ fenalce/ nueva/plantillas/arch_web/APR2015AA5.pdf(con acceso el 16/01/2016).

17. FELLER, C.; BLEIHOLDER, H.; BUHR, L.; HACK H.; HESS, M.; KLOSE, R.; MEIER, U.; STAUSS, R.; VAN DEN BOOM, T.; WEBER, E. 1995. Phänologische Entwicklungsstadien von Gemüsepflanzen:
II. Fruchtgemüse und Hülsenfrüchte. Nachrichtenbl. Deutsch. Pflanzenschutz. 47:217-232.

18. FLOWERS, T.; YEO, A. 1986. Ion relations of plants under drought and salinity. Australian J. Scientific Res. 13(1):75-91.

19. FOOD AND AGRICULTURE ORGANIZATION -FAO-. 2000. Land resource potential and constraints at regional and country levels. Land and Water Development Division. FAO, Rome. 63p.

20. GILI, P.; MARANDO, G.; IRISARRI, J.; SAGARDOY, M. 2004. Efecto de las técnicas de lavado y fertilización sobre la salinidad en suelos del alto valle de río negro y neuquén, Argentina. Agr.Técnica. 64(3):295-304.

21. GOUIA, H.; GHORBAL, M.; TOURAIWE, C. 1994. Effects of $\mathrm{NaCl}$ on flows of $\mathrm{N}$ and mineral ions and $\mathrm{NO}^{-3}$ reductase rate withing whole plant of salt-sensitive bean and tolerant cotton. Plant Physiol. 105:14091418.

22. HARTUNG, W.; SAUTER, A.; HOSE, E. 2002. Abscisic acid in the xylem: where does it comes from, where does it goes to? J. Exp. Bot. 53: 27-32.

23. HASEGAWA, P.M.; BRESSAN, R.; ZHU, J.; BOHNERT, H. 2002. Plant celular and molecular responses to high salinity. Ann. Rev. Plant Physiol. Plant Mol. Biol. 51: 463-499.

24. JARAMILLO, C.; OLIVA, M.; FERREIRA, R. 2009. Respuesta fotosintética de diferentes ecotipo de fríjol a la radiación y la salinidad. Corpoica. Cienc. Tecnol. Agropec. 10(2):129-140.

25. KAYMAKANOVA, M.; STOEVA, N.; MINCHEVA, T. 2008. Salinity and its effects on the physiological response of bean (Phaseolus vulgaris L.). J. Central Eur. Agric. 9(4):749-756.

26. KUMAR, D. 1991. Salt-tolerance of some induced mutants of HD 2009 wheat. Indian J. Agr. Sci. 51(7):475479.

27. LIZANA, C.; WENTWORTH, M.; MARTÍNEZ, J.; VILLEGAS, D.; MENESES, R.; MURCHIE, E.; PASTENES, C.; LECARI, B.; VERNIERI, P.; HORTON, P.; PINTO, M. 2006. Differential adaptation of two varieties of common bean to abiotic stress I. Effect of drought on yield and photosynthesis. J. Exp. Bot. 56: 699709. 
28. MAAS, E. 1990. Crop salt tolerance. In: Tanjii, K. (ed.) Agricultural salinity assessment and management manual. ASCE. New York. p.262-304.

29. MANO, Y.; TAKEDA, K. 2001. Genetic resources of salttolerance at germination and the seedling stage in wheat. Jap. J. Crop Sci. 70(2):215-220.

30. McCUE, K.; HANSON, A. 1990. Drought and salt tolerance, towards understanding and application. Trends Biotechnol. 8: 358-362.

31. MINISTERIO DE AMBIENTE, VIVIENDA Y DESARROLLO TERRITORIAL -MAVDT-. 2004. Plan de acción nacional de lucha contra la desertificación y la sequía en Colombia. MADVT, Bogotá. 33p.

32. RÍOS, M.; QUIRÓS, J. 2002. El frijol su cultivo, beneficio y variedades, edit. Produmedios, Bogotá, D.C, Primera Edición.193p.

33. SUDHIR, P.; MURTHY, S. 2004. Effects of salt on basic process of photosynthesis. Photosynthetica. 42(4):481-486.
34. TABATABAEI, S.J. 2006. Effects of salinity and $N$ on the growth, photosynthesis and $\mathrm{N}$ status of olive (Olea europaea L.) trees. Scientia Horticulturae. 108(4):432-438.

35. VELÁZQUEZ M., M.A; ORTEGA E., M.; MARTÍNEZ G., A.; KOHASHI S., J.; GARCÍA C., N. 2002. Relación funcional PSI-RAS en las aguas residuales y suelos del Valle de Mezquital, Hidalgo. México. Terra Latinoam. 20:459-464.

36. VISWANATHAN, C.; ZHU, J. 2003. Plant salt tolerance. En: Hirt, H.; Schinozaki, K. (eds). Plant responses to abiotic stress. Topics in current genetics. Berlin: Springer-Verlag. p.241-270.

37. ZÚINIIGA, O.; OSORIO, J.; CUERO, R.; PEÑA, J. 2011. Evaluación de tecnologías para la recuperación de suelos degradados por salinidad. Rev. Fac. Nal. Agr. Medellín. 64(1):5769-5779.

Recibido: Enero 12 de 2016

Aceptado: Mayo 23 de 2016

Cómo citar:

Quintana-Blanco, W.A.; Pinzón-Sandoval, E.H.; Torres, D.F. 2016. Evaluación del crecimiento de fríjol (Phaseolus vulgaris L.) Cv ICA Cerinza, bajo estrés salino. Rev. U.D.C.A Act. \& Div. Cient. 19(1): 87-95. 